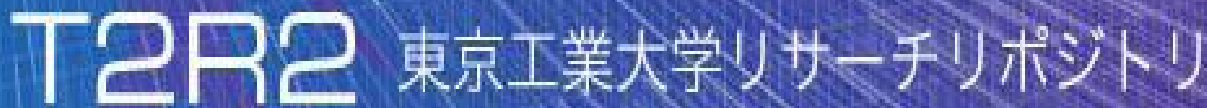

\section{Tokyo Tech Research Repository}

\section{論文 /著書情報 \\ Article /Book Information}

\begin{tabular}{|c|c|}
\hline Title & $\begin{array}{l}\text { Efficient calculation method for realistic deep 3D scene hologram using } \\
\text { orthographic projection }\end{array}$ \\
\hline Authors & $\begin{array}{l}\text { Shunsuke Igarashi, Tomoya Nakamura, Masahiro Yamaguchi, Kyoji } \\
\text { Matsushima }\end{array}$ \\
\hline Citation(English) & Proc. SPIE, Vol. 9771, , 977100,10 pages \\
\hline 発行日 /Pub. date & 2016, 3 \\
\hline DOI & $10.1117 / 12.2212301$ \\
\hline 権利情報 /Copyright & $\begin{array}{l}\text { 本著作物の著作権はSociety of Photo-O ptical Instrumentation } \\
\text { Engineersに帰属します。 } \\
\text { Copyright } 2016 \text { Society of Photo-O ptical Instrumentation Engineers. } \\
\text { One print or electronic copy may be made for personal use only. } \\
\text { Systematic reproduction and distribution, duplication of any material in } \\
\text { this paper for a fee or for commercial purposes, or modification of the } \\
\text { content of the paper are prohibited. }\end{array}$ \\
\hline
\end{tabular}




\title{
Efficient Calculation Method for Realistic Deep 3D Scene Hologram using Orthographic Projection
}

\author{
Shunsuke Igarashi ${ }^{*}$, Tomoya Nakamura ${ }^{\mathrm{a}}$, Kyoji Matsushima ${ }^{\mathrm{b}}$, Masahiro Yamaguchi ${ }^{\mathrm{a}}$ \\ a Department of Information Processing, Interdisciplinary Graduate School of Science and \\ Engineering, Tokyo Institute of Technology, 4259 Nagatsuda-cho, Midori-ku, Yokohama, \\ Kanagawa, 226-8503, Japan; \\ ${ }^{b}$ Department of Electrical and Electronic Engineering, Kansai University, 3-3-35 Yamate-cho, Suita, \\ Osaka 564-8680, Japan
}

\begin{abstract}
We propose a fast calculation method to synthesize a computer-generated hologram (CGH) of realistic deep threedimensional (3D) scene. In our previous study, we have proposed a calculation method of CGH for reproducing such scene called ray-sampling-plane (RSP) method, in which light-ray information of a scene is converted to wavefront, and the wavefront is numerically propagated based on diffraction theory. In this paper, we introduce orthographic projection to the RSP method for accelerating calculation time. By numerical experiments, we verified the accelerated calculation with the ratio of 28-times compared to the conventional RSP method. The calculated CGH was fabricated by the printing system using laser lithography and demonstrated deep 3D image reconstruction in $52 \mathrm{~mm} \times 52 \mathrm{~mm}$ with realistic appearance effect such as gloss and translucent effect.
\end{abstract}

Keywords: computer generated hologram, 3D computer graphics

\section{INTRODUCTION}

Holography is a method that can reproduce 3D image satisfying all depth cues in human perception from an interference fringe called hologram. To synthesize a hologram computationally, a number of techniques for calculating computer generated holograms (CGHs) have been proposed. These techniques are broadly classified into two groups: wavepropagation-based methods [1][2] and light-ray based methods [3][4]. In the wave-propagation-based methods, objects are expressed by a set of self-illuminant point sources or polygons. The CGH generated by such method can reconstruct an accurate and high-resolution image even in deep scene. In recent reports, methods for acceleration of calculation [5] and realization of view-dependent effects [6] have been implemented for polygon-based method; however, the realistic expression of complex optical phenomena such as reflection, texture, and translucence is still difficult problems in wavefront-based rendering. On the other hand, in the light-ray based methods, a hologram can be calculated from images captured by 3D computer graphics (3DCG) or photographs. It can visualize complicated texture and occlusion of objects with using various advanced rendering techniques in 3DCG; however, it sacrifices the image resolution; the image resolution is limited if the reconstructed image is distant from the hologram plane, because of the discontinuity of lightray sampling and the diffraction effect [7][8].

To utilize advantages of these two methods in CGH calculation, we previously proposed the ray-sampling-plane (RSP) method [8][9]. It was verified that the hologram calculated with the RSP method can reproduce high-resolution 3D images even with deep scene maintaining realistic appearance; however, the large computational cost was still a problem. In this paper, orthographic projection is introduced to eliminate the redundant calculation in numerical wavefront propagation in the conventional RSP method. In previous studies, several methods using orthographic projection images have been proposed [10-13]. Sando et al. proposed a method that fills a parabolic surface in the Fourier domain with a Fouriertransformed orthographic captured image [10]. Park et al. also investigated a technique based on orthographic projection [11]. If the depth range of the scene is large, the image resolution is degraded due to the diffraction effect in these methods. Reconfigurable image projection hologram [12] is also a similar technique to the original RSP method, although the method only deal with horizontal-parallax-only (HPO) case. These conventional algorithms have similar characteristics to

*igarashi.s.ab@m.titech.ac.jp; phone +81-045-924-5137; fax +81-045-924-5137

Practical Holography XXX: Materials and Applications, edited by Hans I. Bjelkhagen,

V. Michael Bove, Proc. of SPIE Vol. 9771, 977100 - (c) 2016 SPIE

CCC code: $0277-786 \mathrm{X} / 16 / \$ 18 \cdot$ doi: $10.1117 / 12.2212301$

Proc. of SPIE Vol. $9771977100-1$ 
the method introduced in this paper; however, it is not possible to guarantee the image quality in a deep scene in these methods, while the RSP method allows the reproduction of 3D image visually almost identical to that of real hologram even in a deep scene. Additionally, this framework provides high-performance even in the case of a large-scale full-parallax hologram. This algorithm is mainly maintained by the FFT and simple complex multiplication of array, which are highly optimized for image processing. In this paper, an extremely high-resolution hologram was synthesized by the proposed method in practical computational time. The calculated hologram was fabricated by high-resolution laser lithography system [13], and it was confirmed that the realistic texture, specular reflection, and translucent effect are reproduced by the CGH. Additionally, we verified that our method is useful especially when a large object is assumed at a far distance from the hologram plane.

\section{COMPUTER-GENERATED HOLOGRAM USING RAY-SAMPLING PLANE}

Figure 1 shows the schematic diagram of CGH calculation using the RSP. In the method, at first, the RSP is defined near the object, and the light-ray information of the object is densely sampled. In this paper, a unit image in the sampled lightray information is called as elemental image. In conventional light-ray sampling, each elemental image corresponds to a perspective projection image, which captures light-rays diverging to different angles passing through a single spatial position. The wavefront in the small area around the captured point can be obtained by Fourier transform of the elemental image because each pixel in the elemental image corresponds to a certain direction, or one frequency component of the angular spectrum. The resultant elemental wavefront can be used as a small part of a whole wavefront of the object, thus the whole wavefront can be obtained by aligning all Fourier transformed elemental images. This process can be considered as the conversion of the light-ray information into the wavefront on the RSP. The obtained wavefront near the object plane is propagated to a hologram plane based on diffraction theory, such as Fresnel diffraction or angular spectrum method.

When light-rays are sampled, the diffraction effect affects the image resolution. However, if the rays are sampled near the object, the degradation of resolution by diffraction is small and it can be visually negligible. In addition, the diffractionbased calculation of wave-propagation from the RSP to the hologram plane does not sacrifices the information of object even with deep propagation, thus the resultant hologram can reproduce accurate 3D image if the sampling density of the ray is enough considering sampling theorem. Additionally, light-field-based processing such as occlusion culling [9] and reflectance-property expression can be achieved thanks to the process light-ray sampling. By these advantages, the RSP method is useful for calculating deep and realistic CGH; however, computational cost is still a problem in this method.

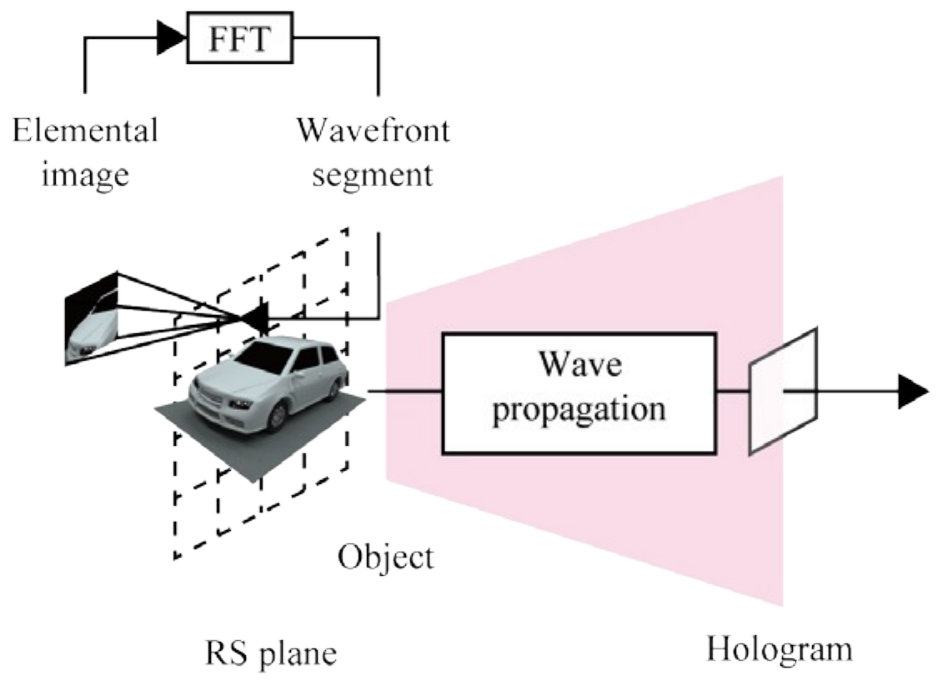

Fig. 1. Schematic diagram of RSP based CGH. 


\section{ACCELERATOIN USING ORTHOGRAPHIC PROJECTION}

As shown in Fig. 2 (a), the generated wavefront from the object contains redundant components which do not incident on the hologram plane. The calculation will be accelerated if such components could be eliminated in calculation; however, it is not easy with the conventional framework of the RS method because each elemental wavefront must diverge within a certain diverging angle.

To solve this problem, the proposed method in this paper uses orthographically sampled images for elemental images instead of perspective images. An orthographic projection image is spatial distribution of parallel light-rays. It can simply be generated by the rearrangement of pixels in the perspective images. In the rearrangement, pixels at the same local coordinates in perspective elemental images are gathered into a new orthographic image.

The concept of conventional and proposed methods are compared in Fig. 2. As shown in Fig. 2 (a), an element in the conventional method contains light rays with different propagation angles. In this case, all elemental images have to be Fourier transformed, thus the removal of the calculation of redundant light-rays cannot be implemented. Contrary, an orthographic image is a collection of parallel light-rays. Therefore, as shown in Fig 2 (b), we can easily separate unnecessary light-rays, which do not incident on the hologram plane. This fact is advantageous in acceleration of the RSP based CGH calculation.

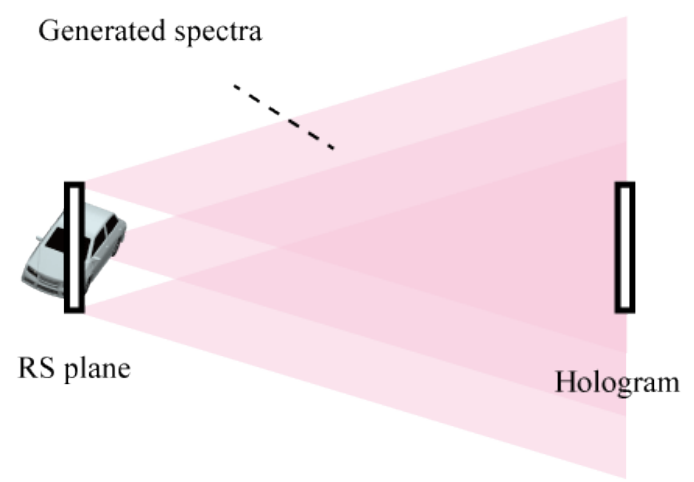

(a)

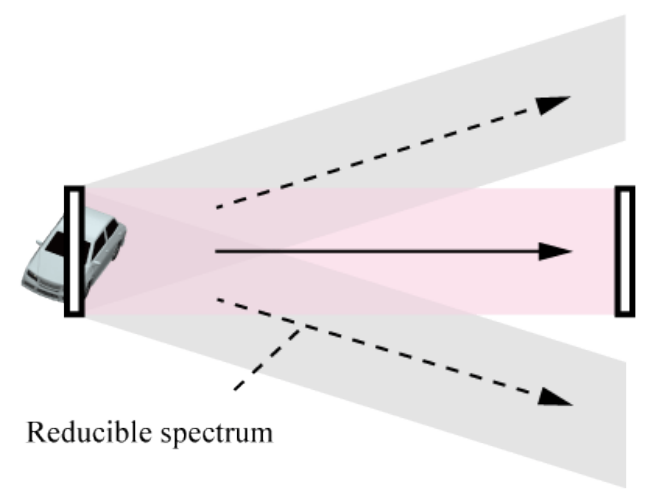

(b)

Fig. 2. Light-ray information in (a) conventional RSP method and (b) proposed method. 
Moreover, orthographic projection physically corresponds to the inclined plane waves, and the wavefront at the RSP can be obtained with the accumulation of the orthographic images multiplied with inclined plane waves. It means that the Fourier transformed RSP is generated from the Fourier transformed orthographic images that are shifted in Fourier domain. This result is directly used for multiplication with a transfer function, which is generally band-limited for preventing aliasing. This also contributes to omission of redundant components of the object wave in calculation. This feature enables us to realize further acceleration of calculation, especially when the RSP is large and located in far distance from the hologram plane.

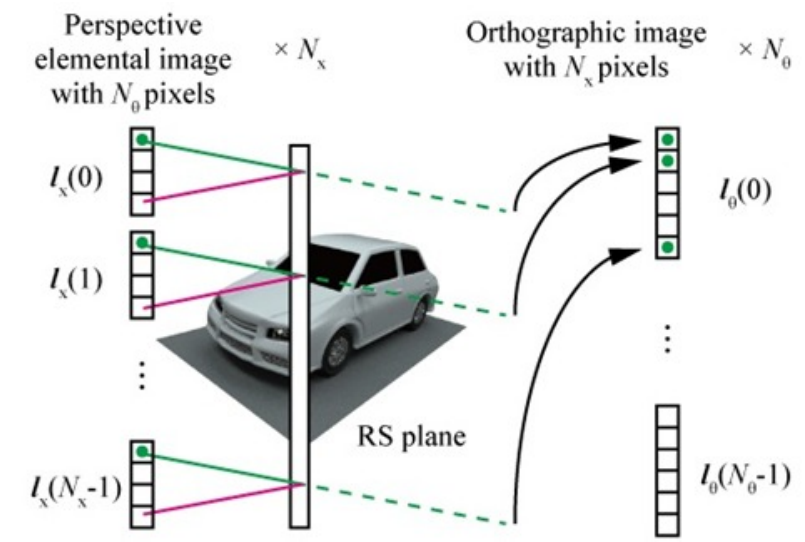

Fig. 3. Relation between perspective and orthographic images.

Figure 3 shows the relation of pixels in a set of perspective elemental images $\boldsymbol{l}_{\mathrm{x}} \in \mathbb{R}^{N_{\theta} N_{\mathrm{x}} \times 1}$ and a set of orthographic images $\boldsymbol{l}_{\theta} \in \mathbb{R}^{N_{\mathrm{x}} N_{\theta} \times 1}$, where $N_{\theta}$ is the length of a vector of the perspective elemental image, $N_{\mathrm{x}}$ is that of the orthographic image, and $\mathbb{R}^{N_{i} \times N_{j}}$ denotes an $N_{i} \times N_{j}$ matrix of real numbers. For simplicity, the y-axis is omitted in the discussion in this paper. An orthographic image is a set of spatially distributed parallel light rays, and thus, pixels at the same local coordinates in perspective elemental images correspond to pixels in a specific orthographic image. The vector of the $n$-th perspective image $\boldsymbol{l}_{\mathrm{x}}(n) \in \mathbb{R}^{N_{\theta} \times 1}$ can be written in expanded form as:

$$
\boldsymbol{l}_{\mathrm{x}}(n)=\left[\begin{array}{c}
\boldsymbol{l}_{\mathrm{x}}(n)[0] \\
\boldsymbol{l}_{\mathrm{x}}(n)[1] \\
\vdots \\
\boldsymbol{l}_{\mathrm{x}}(n)\left[N_{\theta}-1\right]
\end{array}\right]
$$

where $\boldsymbol{l}[\cdot]$ means a vector element. In the orthographic case, the vector of the $m$-th orthographic image $\boldsymbol{l}_{\theta}(m) \in \mathbb{R}^{N_{\mathrm{x}} \times 1}$ and the relation to the perspective images are as follows:

$$
\boldsymbol{l}_{\theta}(m)=\left[\begin{array}{c}
\boldsymbol{l}_{\theta}(m)[0] \\
\boldsymbol{l}_{\theta}(m)[1] \\
\vdots \\
\boldsymbol{l}_{\theta}(m)\left[N_{\mathrm{x}}-1\right]
\end{array}\right]=\left[\begin{array}{c}
\boldsymbol{l}_{\mathrm{x}}(0)[m] \\
\boldsymbol{l}_{\mathrm{x}}(1)[m] \\
\vdots \\
\boldsymbol{l}_{\mathrm{x}}\left(N_{\theta}-1\right)[m]
\end{array}\right]
$$

Note that the perspective elemental image of the light field, $\boldsymbol{l}_{\mathbf{x}}$, contains diverging light rays from a single spatial coordinate, whereas that of $\boldsymbol{l}_{\theta}$ consists of parallel light rays from varying spatial coordinates. 
Figure 4 illustrates a schematic diagram of the wave propagation calculation using the conventional perspective images and proposed orthographic images from the RSP to the hologram plane. To obtain the wavefront at the hologram plane, $\boldsymbol{w}_{\mathrm{h}}$, the wavefront at the RSP, $\boldsymbol{w}_{\mathrm{RS}}$, is diffracted numerically. Here, $\boldsymbol{w}$. denotes complex wavefront vector in the spatial domain. For the diffraction calculation, we adopt the shifted angular spectrum method [14] because of its accuracy and suitability for off-axis propagation. The calculation is modeled as follows:

$$
\boldsymbol{w}_{\mathrm{h}}=\mathcal{F}^{-1}\left[\mathcal{F}\left[\boldsymbol{w}_{\mathrm{RS}}\right] \boldsymbol{H}(u ; z)\right]
$$

where $\mathcal{F}[\cdot]$ is the Fourier transform operator, and $\boldsymbol{H}(u ; z)$ corresponds to the transfer function, which depends on the propagation distance $\mathrm{z}$. In the previous RSP method, $\mathcal{F}\left[\boldsymbol{w}_{R S}\right]$ in Eq. (3) is calculated by

$$
\mathcal{F}\left[\boldsymbol{w}_{\mathrm{RS}}\right]=\mathcal{F}\left[\begin{array}{c}
\mathcal{F}\left[\boldsymbol{l}_{\mathrm{x}}(0)\right] \\
\mathcal{F}\left[\boldsymbol{l}_{\mathrm{x}}(1)\right] \\
\vdots \\
\mathcal{F}\left[\boldsymbol{l}_{\mathrm{x}}\left(\mathrm{N}_{\theta}-1\right)\right]
\end{array}\right] .
$$

On the other hand, the orthographic image physically corresponds to the plane wave with a specific angular spectrum; thus, $\boldsymbol{w}_{\mathrm{RS}}$ can be obtained by integrating $\boldsymbol{l}_{\theta}$ multiplied by inclined plane waves. It means that $\mathcal{F}\left[\boldsymbol{w}_{R S}\right]$ in Eq. (3) can also be obtained just by using the stack of Fourier-transformed orthographic images that are shifted in the Fourier domain.

$$
\mathcal{F}\left[\boldsymbol{w}_{\mathrm{RS}}\right]=\left[\begin{array}{c}
\mathcal{F}\left[\boldsymbol{l}_{\theta}\left(\mathrm{N}_{\mathrm{x}}-1\right)\right] \\
\mathcal{F}\left[\boldsymbol{l}_{\theta}\left(\mathrm{N}_{\mathrm{X}}-2\right)\right] \\
\vdots \\
\mathcal{F}\left[\boldsymbol{l}_{\theta}(0)\right]
\end{array}\right] .
$$

As mentioned above, $\boldsymbol{l}_{\theta}$ in Eq. (5) physically corresponds to plane waves with angular spectra; therefore, the redundant $\boldsymbol{l}_{\theta}(m)$ that does not affect the hologram plane can easily be omitted from the calculation of Eq. (5) in advance. In addition to the above discussion, the transfer function $\boldsymbol{H}(u ; z)$ is band-limited for preventing aliasing. These features allow the calculation of Eq. (5) to be compressed. As predicted by the characteristics, the achievable compression ratio of the calculation time becomes larger when the size of or distance to the virtual object is large.

As explained above, this algorithm consists of FFT and complex multiplications. This feature enables us to use highly optimized libraries for each computational environment. Parallel computing is also able to be implemented by parallelizing FFT and multiplication for each orthographic image. 


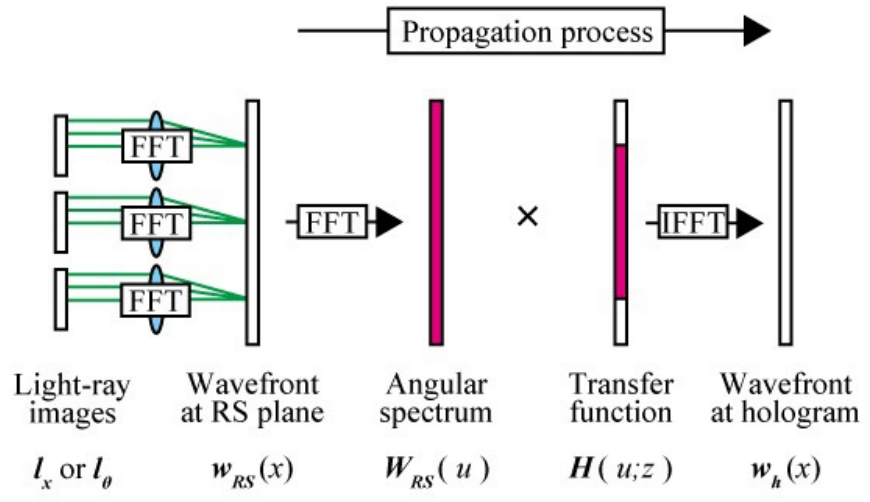

(a)

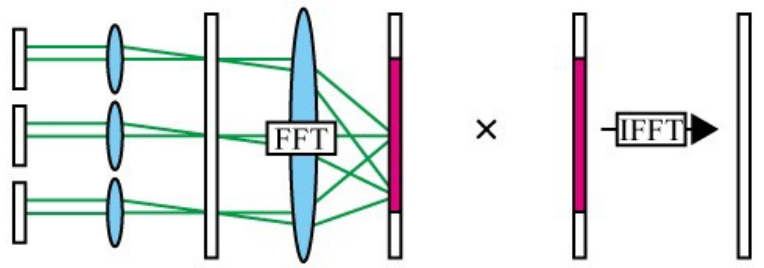

(b)

Fig. 4. Flow of (a) conventional and (b) proposed method for synthesizing hologram using ray-wavefront conversion.

\section{EXPERIMENTAL VERIFICATION}

In the first experiment, we verified the effect of acceleration by the proposed method. In this experiment, the spatial resolution of the hologram was fixed to $2048 \times 2048$ pixels, and the resolution of the RSP was changed from $2048 \times$ 2048 pixels to $16384 \times 16384$ pixels. Other parameters are shown in Table 1 .

Table 1. Parameters used for measuring the calculation time.

\begin{tabular}{c|c}
\hline Wavelength & $532 \mathrm{~nm}$ \\
Pixel pitch & $2 \mu \mathrm{m}$ \\
Propagation distance & $0.30 \mathrm{~m}$ \\
Number of orthographic images & $16 \times 16$ \\
\hline
\end{tabular}




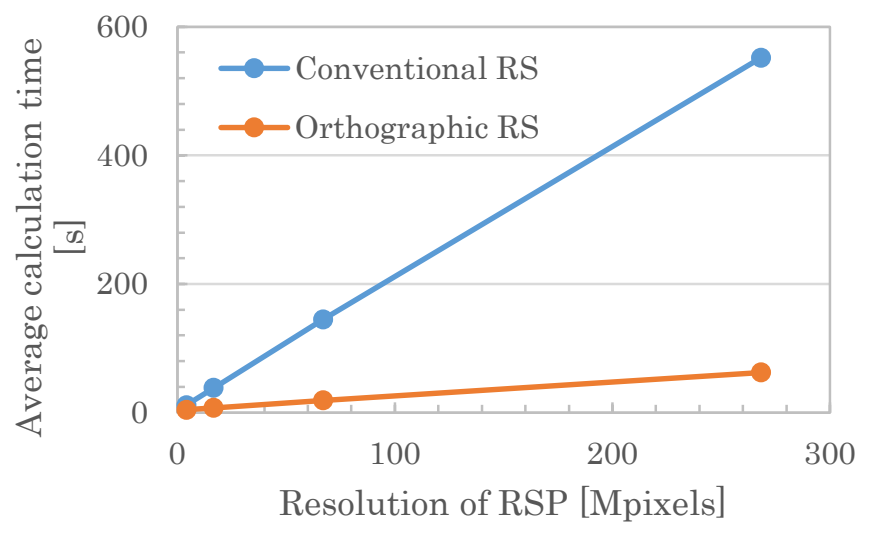

Fig. 5. Relation between calculation time and the size of RSP.

The average calculation time to synthesize hologram is shown in fig. 5 with an Intel Core i7 4790K CPU 4.0 GHz and 32GB RAM. As indicated in the figure, the acceleration ratio between the proposed and conventional methods was 9-times over the varying sizes of the RSP. From this result, we concluded that the proposed method is effective for the fast calculation of the RSP based CGH, especially when we assume large objects placed at deep distance from the hologram plane.

In the second experiment, we experimentally verified that our method does not lose the resolution of the reconstructed image even with deep scene. Parameters used in this experiment are shown in Table 2. The locations of the object, the RSP, and the hologram are depicted in Fig. 6. We created a 3D scene with a stuffed bear, wooden floor and a glass of white wine. This scene includes complicated textures: the shaded wool and the wood. Moreover, light reflection and refraction by wine in a glass is difficult to be computed by wave-propagation-based CGH. Orthographic images of this scene were rendered in different $64 \times 64$ angles by 3DCG software Blender. The time required for the rendering of orthographic images was around 2 minutes.

The calculation time for synthesizing this hologram from the orthographic images was 147 minutes, which corresponds to around 28-times acceleration compared with the conventional RSP-based method, which can be estimated from Fig. 5. Calculated wavefront at the hologram plane was converted to binary fringe pattern by calculating the interference with a reference wave, spherical wave in this case.

Generated binary fringe pattern was saved as BMP data. This image data was transferred to Kan-Dai Digital HoloStudio [15][16], in which a CGH was printed by a laser lithography system (Heidelberg Instruments DWL 66+). The specification of this system is shown in Table 3. For printing the CGH by the laser lithography system, the BMP image data was converted to a CAD data. The pixel pitch of the printed CGH was $0.8 \mu \mathrm{m}$, which corresponds to $\pm 23.3^{\circ}$ field of view when reconstructed by $633 \mathrm{~nm}$ wavelength light.

Table 2. Parameters used in 3D image reconstruction.

\begin{tabular}{c|c}
\hline Resolution of hologram & $65536 \times 65536$ pixels \\
Resolution of RSP & $65536 \times 65536$ pixels \\
Number of orthographic images & $64 \times 64$ \\
Resolution of an orthographic image & $1024 \times 1024$ pixels \\
Wavelength & $633 \mathrm{~nm}$ \\
Pixel pitch & $0.8 \mu \mathrm{m}$ \\
Propagation distance & $0.12 \mathrm{~m}$ \\
\hline
\end{tabular}




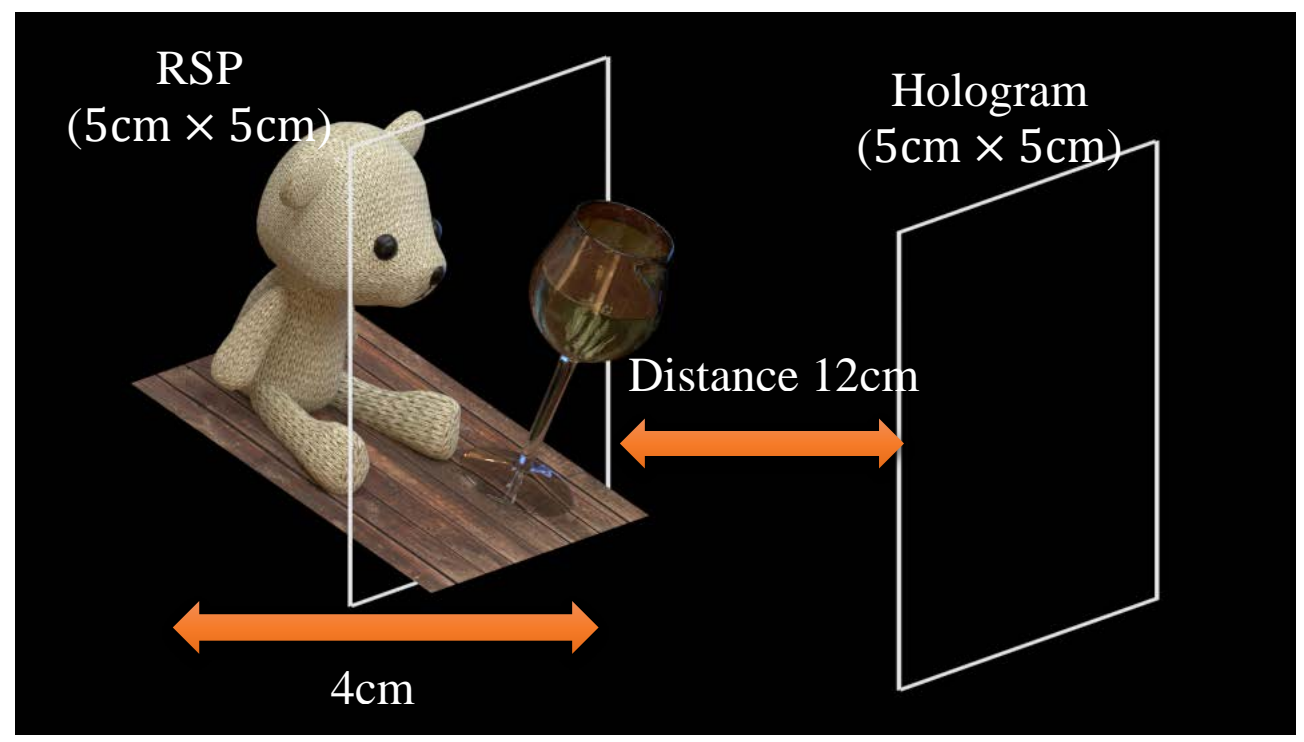

Fig. 6. Geometrical parameters of object, RSP, and hologram.

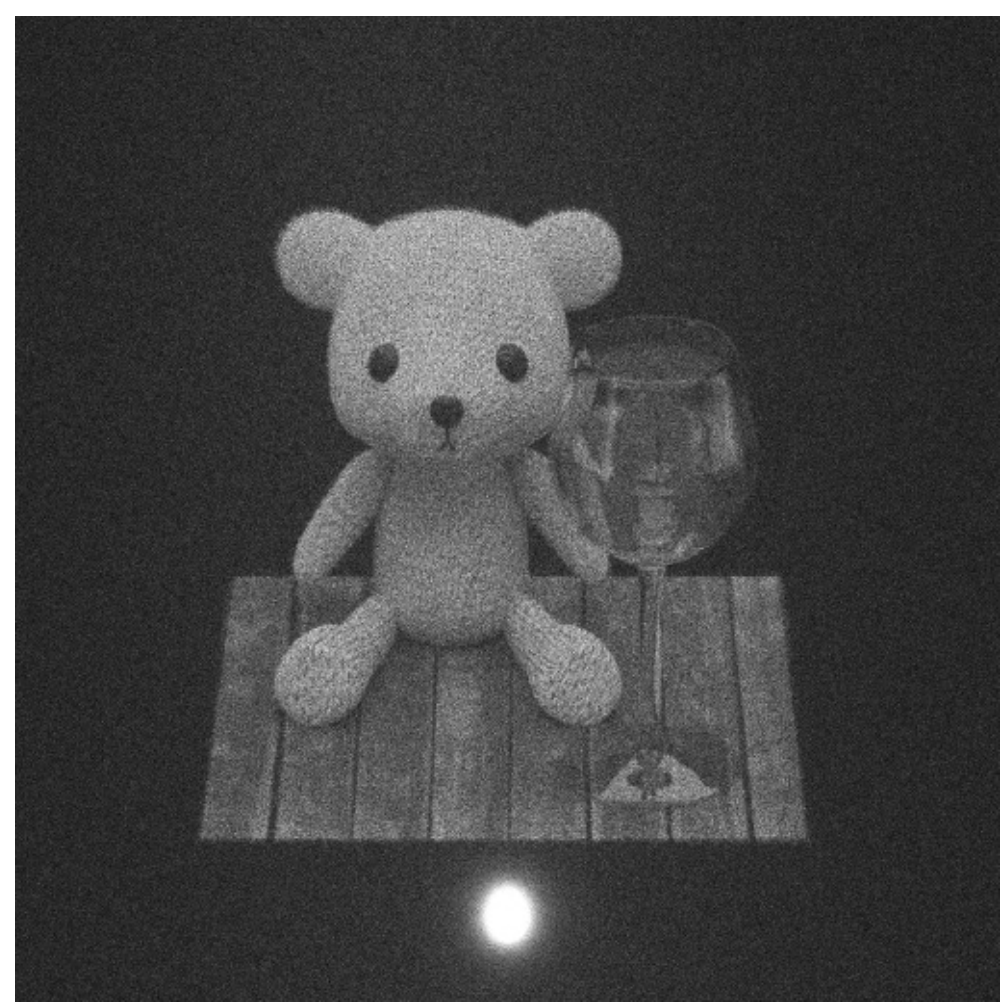

Fig. 7. 3D image reconstructed from the synthesized hologram using proposed method.

Proc. of SPIE Vol. $9771977100-8$ 


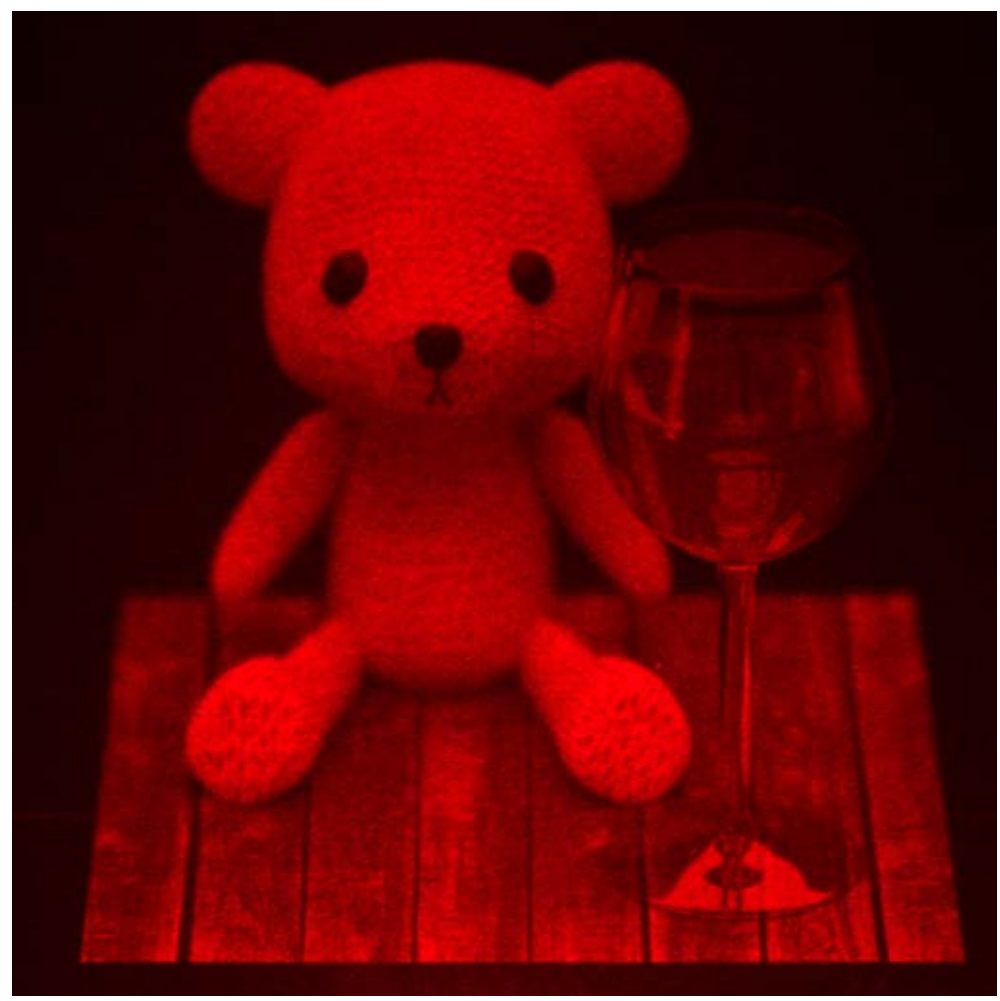

(a)

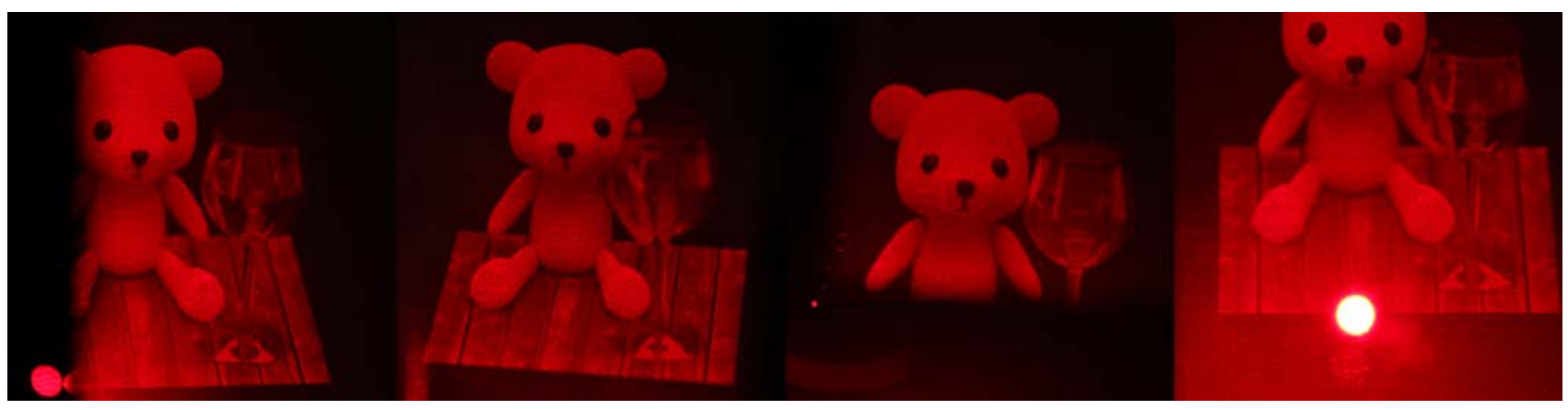

(b)

Fig. 8. Optical reconstruction of the 3D image from the CGH fabricated by the system using laser lithography. (a) Front view and (b) some different angles.

The results of numerical and optical reconstructions are shown in Fig. 7 and Fig. 8, respectively. The CGH was optically reconstructed by the transmitted illumination of spherical wave from He-Ne laser (633nm). As shown in these figures, the optical reconstruction has high image-quality at the same level as numerical one. Thanks to ray-wavefront conversion, the complicated expressions were successfully reproduced in the reconstructed image, and the resolution was still high even with the deep scene as shown in the figures. 


\section{CONCLUSION}

We proposed a fast calculation method to synthesize CGH of realistic deep 3D scene hologram based on ray-wavefront conversion and orthographic projection. In the proposed method, orthographic projection is utilized to separate redundant light-waves, which do not incident on the hologram plane. By numerical experiments, the effect of acceleration in CGH calculation was experimentally evaluated, and the 3D image reconstruction with the CGH including the large and deep objects was demonstrated. In the demonstration, the calculated CGH was recorded by the printing system using laser lithography and demonstrated deep 3D image reconstruction. The large and deep 3D scene containing complicated texture, light reflection, and refraction was successfully reproduced with the practical computational time for calculating the corresponding hologram.

\section{ACKNOWLEDGEMENT}

This work was supported by the MEXT strategic research foundation at private universities (2013-2017) and Kan-Dai Holo-Studio in Kansai University.

\section{REFERENCES}

[1] J. P. Waters, "Holographic image synthesis utilizing theoretical methods," Appl. Phys. Lett. 9(11), 405-406 (1966).

[2] K. Matsushima, "Computer-generated holograms for three-dimensional surface objects with shade and texture," Appl. Opt. 44(22), 4607-4614 (2005).

[3] T. Yatagai, "Stereoscopic approach to 3-D display using computer-generated holograms,” Appl. Opt. 15(11), 27229 (1976).

[4] M. Yamaguchi, H. Hoshino, T. Honda and N. Ohyama, "Phase-added stereogram: calculation of hologram using computer graphics technique,” Proc. SPIE 1914, 25-31 (1993).

[5] J. Weng, et al. "Generation of real-time large computer generated hologram using wavefront recording method," Opt. Express, 20(4), 4018-4023 (2012).

[6] H. Nishi, K. Matsuhsima and N. Nakahara, "Rendering of specular surfaces in polygon-based computer-generated holograms,” Appl. Opt. 50(34), 245-252 (2011).

[7] F. Jin, J. S. Jang and B. Javidi, "Effects of device resolution on three-dimensional integral imaging,” Opt. Lett. 29(12), 1345-1347 (2004).

[8] K. Wakunami and M. Yamaguchi, "Calculation for computer generated hologram using ray-sampling plane,” Opt. Exp. 19(10), 9086-9101 (2011).

[9] K. Wakunami, H. Yamashita, and M. Yamaguchi, "Occlusion culling for computer generated hologram based on raywavefront conversion," Opt. Express 21(19), 21811-21822 (2013).

[10] Y. Sando, M. Itoh and T. Yatagai, "Holographic three-dimensional display synthesized from three-dimensional Fourier spectra of real existing objects,” Opt. Lett. 28(24), 2518-2520 (2003).

[11] J. H. Park, M. S. Kim, G. Baasantseren and N. Kim, "Fresnel and Fourier hologram generation using orthographic projection images,” Opt. Express, 17(8), 6320-6334 (2009).

[12] W. Plesniak, M. Halle, V. M. Bove, Jr., J. Barabas and R. Pappu, “Reconfigurable image projection holograms,” Opt. Eng. 45(11), 115801 (2006).

[13]K. Matsushima, M. Nakamura, and S. Nakahara, "Silhouette method for hidden surface removal in computer holography and its acceleration using the switch-back technique,” Opt. Express, 22(20), 24450 - 24465 (2014)

[14] K. Matsushima, "Shifted angular spectrum method for off-axis numerical propagation," Opt. Express, 18(17), 1845318463 (2010).

[15] K. Matsushima, S. Nakahara, S. Masuda, T. Miyaoka, "Research Facilities for Computer Holography at Kansai University and Several Recent Topics," International Workshop on Holography and Related Technologies 2014 (IWH2014), Beijing, china, 16b01, 58-59(2014).

[16] “Kan-Dai Digital Holo-Studio,” http://holography.ordist.kansai-u.ac.jp/digitalholostudio/ 